\title{
Retrospective review of safety and efficacy of programmed cell death-1 inhibitors in refractory high grade gliomas
}

\author{
Samantha N. Reiss ${ }^{1 *}$, Prakirthi Yerram¹', Lisa Modelevsky ${ }^{1}$ and Christian Grommes ${ }^{2}$
}

\begin{abstract}
Background: Programmed cell death ligand-1 (PD-L1) expression has been reported in up to 61\% of high grade gliomas (HGG). The purpose of this study was to describe safety and efficacy of PD-1 inhibition in patients with refractory HGGs.

Methods: This Institutional Review Board approved single center retrospective study included adult patients with pathologically confirmed HGG who received a PD-1 inhibitor from 9/2014-10/2016 outside of a clinical trial at Memorial Sloan Kettering Cancer Center.

Results: Twenty five HGG patients received pembrolizumab as part of a compassionate use program. Median age was 50 years (range 30-72); 44\% were men; 13 had glioblastoma (52\%), 7 anaplastic astrocytoma (28\%), 2 anaplastic oligodendroglioma (8\%), 2 unspecified HGG (8\%), and 1 gliosarcoma (4\%). Median prior lines of treatments were 4 (range 1-9). Nineteen (76\%) previously failed bevacizumab. Median KPS was 80 (range 50-100). Concurrent treatment included bevacizumab in 17 (68\%) or bevacizumab and temozolomide in 2 (8\%) patients. Median number of doses administered was 3 (range 1-14). Outcomes were assessed in 24 patients. PD-1 inhibitor related adverse events included LFT elevations, hypothyroidism, diarrhea, myalgias/arthralgias, and rash. Best radiographic response was partial response $(n=2)$, stable disease $(n=5)$, and progressive disease $(n=17)$. Median progression free survival (PFS) was 1.4 months (range 0.2-9.4) and median overall survival (OS) was 4 months (range $0.5-13.8$ ). Three-month PFS was $12 \%$ and 6 -month OS was $28 \%$.
\end{abstract}

Conclusion: While response rates are low, a few patients had a prolonged PFS. Pembrolizumab was tolerated with few serious toxicities, even in patients receiving concomitant therapy.

Keywords: Glioblastoma, Immune checkpoint, High-grade glioma, PD-1, PD-L1, Pembrolizumab

\section{Background}

High grade malignant gliomas, including anaplastic oligodendrogliomas, anaplastic astrocytoma (grade III) and glioblastomas (grade IV), are the most common primary malignant brain tumors diagnosed in adults [1]. Despite advancements in understanding the underlying pathogenesis, overall survival remains limited with a median survival for glioblastoma, the most aggressive high grade glioma (HGG), between 16 and 19 months [1]. Upfront therapy for glioblastoma consists of maximal safe resection followed by radiation with concurrent temozolomide and adjuvant temozolomide [2]. Median survival for patients with recurrent

\footnotetext{
* Correspondence: reisss@mskcc.org

${ }^{1}$ Department of Pharmacy, Memorial Sloan Kettering Cancer Center, 1275

York Ave, New York, NY 10065, USA

Full list of author information is available at the end of the article
}

grade III and grade IV tumors is 39 and 30 weeks, respectively [3]. Progression free survival at 26 weeks is $28 \%$ for grade III tumors and $16 \%$ for grade IV tumors. Nonsurgical treatment options for recurrent or progressive high grade gliomas are limited. FDA approved treatment options for recurrent glioblastoma include an anti-vascular endothelial growth factor (VEGF) agent, bevacizumab, and low-intensity alternating electric fields (TTFields); neither treatment has been shown to significantly improve overall survival [4-6]. Other treatment options include conventional chemotherapy such as temozolomide in different dosing schedules, carboplatin, irinotecan, and nitrosoureas [7].

Checkpoint inhibitors have advanced treatment for metastatic melanoma, non-small cell lung cancer, renal cell carcinoma, Non-Hodgkin Lymphoma and other malignancies $[8,9]$. For patients diagnosed with non-small 
cell lung cancer, the level of programmed cell death ligand-1 (PD-L1) expression has been associated with improved outcomes to PD-1 inhibitors $[8,10,11]$. The presence of tumor infiltrating lymphocytes and PD-L1 expression has been reported in up to $61 \%$ of high grade gliomas and therefore this checkpoint is a viable target for treatment $[12,13]$. PD-1 inhibitors block the interaction between PD-L1 and its receptor thereby overcoming T-cell inhibition and promoting an immune response against the tumor. Developing effective treatment options for malignant high grade gliomas has proven difficult due to the inability of many medications to cross the blood brain barrier. Data evaluating the penetration of checkpoint inhibitors across the blood brain barrier is limited. However, the activity of immunotherapy for brain metastasis from melanoma and lung cancer has been reported and is promising [14]. Additionally, there have been case reports of prolonged response after checkpoint inhibitors in patients with glioblastoma $[15,16]$. Currently, there are an abundance of clinical trials evaluating checkpoint inhibitors of patients with glioblastoma. Unfortunately, many patients with high grade gliomas are excluded due to previous treatments, performance status, or tumor histology [12, 17, 18]. At our institution, many patients with high grade gliomas that do not qualify for clinical trial receive off label checkpoint inhibitors. The purpose of this retrospective study is to describe efficacy and safety of PD-1 inhibitors in patients with refractory malignant high grade gliomas.

\section{Methods}

\section{Study design}

This was an Institutional Review Board approved singlecenter observational retrospective study performed at Memorial Sloan Kettering Cancer Center evaluating patients with pathology confirmed high grade malignant glioma who received a PD-1 inhibitor outside of a clinical trial. Patients were identified through the pharmacy database and electronic medical records. Inclusion criteria consisted of patients who were 18 years of age or older and had received a PD-1 inhibitor between September 2014 and October 2016. Patients were excluded if they received a PD-1 inhibitor as part of a clinical trial.

\section{Endpoints and assessments}

The primary objective of this study was to describe overall response rate (ORR) on contrast enhanced MRI. Secondary objectives included characterizing toxicities according to the Common Terminology Criteria for Adverse Events (CTCAE) version 4.03 as well as describing progression free survival (PFS) and overall survival (OS). Frequencies and percentages were used to describe categorical variables and medians and ranges were used to describe continuous variables. Kaplan-Meier methods were used to visualize PFS and OS; patients were censored at the last follow up date if an event did not occur.

\section{Results \\ Patient characteristics}

Twenty-nine neuro-oncology patients received a PD-1 inhibitor between September 2014 and October 2016. Four patients were excluded; 3 patients received previous checkpoint inhibitor therapy as part of a clinical trial and 1 patient did not have a high grade glioma. Baseline characteristics are described in Table 1 . The median age was 49 years (range: $30-72$ years), 11 patients were male (44\%), and the majority of patients were Caucasian (88\%). All patients received pembrolizumab as PD-1 inhibitor for treatment of HGG through a compassionate use program. Thirteen patients had pathology confirmed glioblastoma (52\%), 7 anaplastic astrocytoma (28\%), 2 anaplastic oligodendroglioma (8\%), 2 unspecified HGG (8\%), and 1 gliosarcoma (4\%). Four patients (16\%) were MGMT methylated, $12(48 \%)$ were MGMT unmethylated and 9 (36\%) were unknown. Ten patients (40\%) had tumors that harbored an IDH1 mutation, 9 (36\%) were IDH1 wild type, and 6 (24\%) were unknown. Median mutational load was 7 with a range of 3-58 (Table 2). None of the patients were considered to have a hypermutator phenotype, defined as 100 or more mutations, by MSK-Impact. ${ }^{16}$ Patients were heavily pretreated, receiving a median of 4 prior lines of therapy (range 1-9) and 19 patients $(76 \%)$ previously progressed on bevacizumab treatment. Median KPS at initiation of pembrolizumab was 80 (range 50-100). Concurrent treatment with pembrolizumab included bevacizumab in 17 (68\%) or bevacizumab and temozolomide in $2(8 \%)$ patients. Out of the 19 patients who previously failed bevacizumab, 17 continued on bevacizumab with pembrolizumab therapy. Of the six patients who did not previously receive bevacizumab therapy, two were started on bevacizumab in combination with pembrolizumab. Median number of doses of pembrolizumab administered was 3 (range 1-14). Fourteen patients (56\%) were on dexamethasone during their first treatment dose and $19 \mathrm{pa}-$ tients $(79 \%)$ received dexamethasone at some point during the course of treatment with pembrolizumab. Out of the 105 total doses of pembrolizumab administered, 34 doses (32\%) were administered with concomitant dexamethasone for treatment of disease related neurologic symptoms.

\section{Efficacy}

Treatment response and toxicity was evaluable in 24 patients. One patient was excluded from evaluation of response and toxicity because they transitioned to hospice less than one week after their first and only dose of pembrolizumab; therefore, imaging and toxicity data is not available. This patient was included in survival analysis. Best radiographic response was partial response $(n=2,8 \%)$, stable disease $(n=5,21 \%)$, and progressive disease $(n=17,71 \%)$ (Table 3). Both of the 
Table 1 Baseline Characteristics

\begin{tabular}{|c|c|}
\hline Characteristic & $\begin{array}{l}\text { All patients } \\
(n=25)\end{array}$ \\
\hline Age, year (range) & $49(30-72)$ \\
\hline Gender: male, no. (\%) & $11(44)$ \\
\hline \multicolumn{2}{|l|}{ Race } \\
\hline Caucasian, no. (\%) & $22(88)$ \\
\hline Asian, no. (\%) & $1(4)$ \\
\hline Black, no. (\%) & $0(0)$ \\
\hline Latino/Hispanic, no. (\%) & $1(4)$ \\
\hline Other, no. (\%) & $1(4)$ \\
\hline \multicolumn{2}{|l|}{ Diagnosis } \\
\hline Glioblastoma, no. (\%) & $13(52)$ \\
\hline Anaplastic astrocytoma, no. (\%) & $7(28)$ \\
\hline Anaplastic oligodendroglioma, no. (\%) & $2(8)$ \\
\hline Unspecified high grade glioma, no. (\%) & $2(8)$ \\
\hline Gliosarcoma, no. (\%) & $1(4)$ \\
\hline Performance status, KPS (range) & $80(50-100)$ \\
\hline Number of prior therapies, median (range) & $4(1-9)$ \\
\hline Previously received bevacizumab, no. (\%) & $19(76)$ \\
\hline \multicolumn{2}{|l|}{ MGMT status } \\
\hline Methylated, no. (\%) & $4(16)$ \\
\hline Unmethylated, no. (\%) & $12(48)$ \\
\hline Unknown, no. (\%) & $9(36)$ \\
\hline \multicolumn{2}{|l|}{ IDH1 Status } \\
\hline IDH1 Mutated, no. (\%) & $10(40)$ \\
\hline IDH1 Wild Type, no. (\%) & $9(36)$ \\
\hline Unknown, no. (\%) & $6(24)$ \\
\hline Number of mutations by MSK-Impact, median (range) & $7(3-58)$ \\
\hline \multicolumn{2}{|l|}{ PD-1 inhibitor } \\
\hline Pembrolizumab, no. (\%) & $25(100)$ \\
\hline Number of doses administered, median (range) & $3(1-14)$ \\
\hline \multicolumn{2}{|l|}{ Concomitant therapy } \\
\hline Pembrolizumab monotherapy, no. (\%) & $6(24)$ \\
\hline Bevacizumab, no. (\%) & $17(68)$ \\
\hline Cytotoxic chemotherapy + bevacizumab, no. (\%) & $2(8)$ \\
\hline Receiving dexamethasone at time of first dose, no. (\%) & $14(56 \%)$ \\
\hline
\end{tabular}

patients with a partial response received concomitant bevacizumab, and one patient was bevacizumab-naïve. These two patients received pembrolizumab plus bevacizumab in the second and third line setting for treatment of glioblastoma and anaplastic astrocytoma, respectively. Both patients received dexamethasone for management of disease related symptoms, one at initiation of pembrolizumab treatment. Duration of therapy, best radiographic response, previous bevacizumab, and concomitant bevacizumab can be visualized in Figs. 1 and 2. Two patients had stable disease greater than 200 days. One of these patients received bevacizumab plus pembrolizumab after failing 9 prior treatments including bevacizumab containing regimens. The other patient received pembrolizumab monotherapy after failing 2 prior lines of therapy. The first patient was on dexamethasone only during their first dose of pembrolizumab. The second patient did not receive dexamethasone during treatment with pembrolizumab. Of note, 7 of the 18 patients without a clinical response did not require steroids at treatment initiation. The median mutation load was 6 in patients with partial response and stable disease compared to 7 in those who did not respond. Median progression free survival (PFS) was 1.4 months (range 0.2-9.4) and median overall survival (OS) was 4 months (range 0.5-13.8) (Fig. 3). Six month PFS was $12 \%$ and 6 month OS was $28 \%$.

\section{Toxicity}

All toxicities are listed in Table 4. The most common adverse events reported were fatigue (grade $3-4$ : $4 \%$; grade 1-2: $75 \%$ ), headache (grade 3-4: 4\%; grade 1-2: 43\%), nausea (grade 3-4: 4\%; grade 1-2: 37.5\%), diarrhea (grade 3-4: 0\%; grade 1-2: 17\%), seizures (grade 3-4: 4\%; grade 1-2: 17\%), vomiting (grade 3-4: 4\%; grade 1-2: 17\%), myalgias/arthralgia (grade 3-4: 0\%; grade 1-2: $13 \%$ ), and rash (grade 3-4: $0 \%$; grade $1-2: 8 \%$ ). The most common laboratory abnormalities recorded were hyperglycemia (grade 1-2: 79\%), thrombocytopenia (grade 1-2: 50\%), leukopenia (grade 1-2: 37.5\%), ALT elevations (grade 1-2: 33\%), AST elevations (grade 1-2: 29\%), bilirubin elevations (grade 1-2: 21\%), neutropenia (grade 1-2: 21\%), and hypothyroidism (grade 1-2: $17 \%)$. Additionally, $74 \%$ of patients $(n=14)$ who experienced hyperglycemia were receiving dexamethasone. One patient with a history of epilepsy was admitted for a grade 3 seizure. The second patient who experienced grade 3 adverse events, specifically nausea, vomiting, and headache, was admitted for symptoms of increased intracranial pressure due to pathology confirmed recurrent glioblastoma. Lastly, one patient experienced grade 4 cerebral edema requiring emergent surgery 7 days after their first and only dose of pembrolizumab. Pathology confirmed edema was due to rapid tumor progression. No patients discontinued pembrolizumab due to toxicity.

\section{Discussion}

Our study demonstrated that heavily pretreated patients with malignant high grade gliomas have low response rates to pembrolizumab. To our knowledge, this is the first study to investigate PD-1 inhibition in grade III gliomas. Garber and colleagues found that PD-L1 expression was only present on grade IV gliomas, where as it was not present in the 33 anaplastic astrocytomas or 9 oligodendrogliomas. [19] There is no current data correlating PD-L1 expression and clinical outcomes outside of pembrolizumab use in non-small cell lung cancer. In our grade III glioma cohort, 1 patient had a partial response to pembrolizumab and 2 
Table 2 Patient Characteristics, Response and Steroid Dose

\begin{tabular}{|c|c|c|c|c|c|c|c|c|c|c|c|c|c|c|c|}
\hline \multirow[t]{2}{*}{$\begin{array}{l}\mathrm{Pt} \\
\#\end{array}$} & \multirow[t]{2}{*}{ Grade } & \multirow[t]{2}{*}{ OR } & \multirow[t]{2}{*}{ KPS } & \multirow[t]{2}{*}{$\begin{array}{l}\text { \# of cycles } \\
\text { of pembro }\end{array}$} & \multirow[t]{2}{*}{ MGMT Status } & \multirow[t]{2}{*}{$\begin{array}{l}\text { IDH } \\
\text { Status }\end{array}$} & \multirow[t]{2}{*}{$\begin{array}{l}1 p / \\
19 q\end{array}$} & \multirow[t]{2}{*}{$\mathrm{ML}$} & \multirow[t]{2}{*}{$\begin{array}{l}\text { Steroids at } \\
\text { initiation }\end{array}$} & \multicolumn{3}{|c|}{$\begin{array}{l}\text { Steroid dose at initiation } \\
\text { (in prednisone equivalence) }\end{array}$} & \multirow[t]{2}{*}{$\begin{array}{l}\text { \# of cycles } \\
\text { with steroids }\end{array}$} & \multirow[t]{2}{*}{$\begin{array}{l}\text { Con } \\
\text { Bev }\end{array}$} & \multirow[t]{2}{*}{$\begin{array}{l}\text { Prev } \\
\text { Bev }\end{array}$} \\
\hline & & & & & & & & & & 0 & $<20$ & $\geq 20$ & & & \\
\hline 1 & III & $P R$ & 90 & 4 & unmethylated & WT & N/A & 6 & $\mathrm{~N}$ & $x$ & & & 2 & Y & Y \\
\hline 2 & IV & $P R$ & 80 & 4 & unmethylated & N/A & N/A & 6 & Y & & & $x$ & 1 & Y & $\mathrm{N}$ \\
\hline 3 & III & SD & 90 & 14 & methylated & WT & intact & 6 & $\mathrm{~N}$ & $x$ & & & 0 & $\mathrm{~N}$ & $\mathrm{~N}$ \\
\hline 4 & III & SD & 70 & 10 & unmethylated & MUT & N/A & 3 & Y & & & $x$ & 1 & Y & Y \\
\hline 5 & IV & SD & 90 & 14 & unmethylated & WT & N/A & 12 & $\mathrm{~N}$ & $x$ & & & 5 & Y & Y \\
\hline 6 & IV & SD & 100 & 4 & methylated & N/A & N/A & 13 & $\mathrm{~N}$ & $x$ & & & 0 & $\mathrm{~N}$ & Y \\
\hline 7 & IV & SD & 100 & 1 & N/A & WT & N/A & N/A & Y & & & $x$ & 1 & Y & Y \\
\hline 8 & III & PD & 90 & 6 & $\mathrm{~N} / \mathrm{A}$ & MUT & co-del & 5 & Y & & $x$ & & 1 & N & $\mathrm{N}$ \\
\hline 9 & III & PD & 60 & 5 & N/A & MUT & co-del & 58 & Y & & $x$ & & 0 & Y & Y \\
\hline 10 & III & PD & 60 & 3 & unmethylated & MUT & $\mathrm{N} / \mathrm{A}$ & 7 & $\mathrm{~N}$ & $x$ & & & 2 & N & $\mathrm{N}$ \\
\hline 11 & III & PD & 70 & 3 & unmethylated & WT & N/A & 5 & Y & & $x$ & & 3 & Y & Y \\
\hline 12 & III & PD & 90 & 2 & unmethylated & WT & intact & 15 & Y & & $x$ & & 2 & Y & $\mathrm{N}$ \\
\hline 13 & III & PD & 90 & 2 & unmethylated & MUT & N/A & 7 & Y & & & $x$ & 2 & Y & Y \\
\hline 14 & III & PD & N/A & 1 & methylated & MUT & N/A & 5 & $\mathrm{~N}$ & $x$ & & & 0 & $\mathrm{~N}$ & Y \\
\hline 15 & IV & PD & 90 & 5 & methylated & MUT & N/A & 11 & Y & & & $x$ & 2 & $\mathrm{~N}$ & $\mathrm{~N}$ \\
\hline 16 & IV & PD & 60 & 5 & unmethylated & WT & intact & 10 & Y & & & $x$ & 2 & Y & Y \\
\hline 17 & IV & PD & 50 & 4 & N/A & N/A & N/A & N/A & $\mathrm{N}$ & $x$ & & & 0 & Y & Y \\
\hline 18 & IV & PD & 90 & 3 & N/A & MUT & N/A & 9 & Y & & & $x$ & 3 & Y & Y \\
\hline 19 & IV & PD & 90 & 3 & unmethylated & N/A & N/A & 4 & Y & & & $x$ & 1 & Y & Y \\
\hline 20 & IV & PD & 90 & 3 & N/A & N/A & N/A & N/A & $\mathrm{N}$ & $x$ & & & 1 & Y & Y \\
\hline 21 & IV & PD & 70 & 2 & unmethylated & WT & $\mathrm{N} / \mathrm{A}$ & 13 & Y & & $x$ & & 2 & Y & Y \\
\hline 22 & IV & PD & 80 & 2 & unmethylated & MUT & N/A & 5 & $\mathrm{~N}$ & $x$ & & & 0 & Y & Y \\
\hline 23 & IV & PD & 80 & 2 & N/A & WT & N/A & N/A & $\mathrm{N}$ & $x$ & & & 1 & Y & Y \\
\hline 24 & N/A & PD & 70 & 2 & N/A & N/A & N/A & N/A & $\mathrm{N}$ & $x$ & & & 1 & Y & Y \\
\hline 25 & IV & N/A & 60 & 1 & N/A & MUT & intact & 19 & Y & & & $x$ & 1 & Y & Y \\
\hline
\end{tabular}

Abbreviations: Pt: Patient; OR: Objective response; CR: Complete response; PR: Partial response; SD: Stable disease; PD: Progressive disease; KPS: Karnofsky performance score; Pembro: pembrolizumab; N/A: not applicable or unknown; MGMT methylated: methylated; MGMT unmethylated: unmethylated; IDH mutant: MUT; IDH wild type: WT; 1p19q intact: intact; 1p19q codeleted: Co-del; ML: mutational load by MSK impact; Y: yes; N: no; X: indicates steroid dose at initiation; Con Bev: Concomitant bevacizumab; Prev Bev: previously progressed on bevacizumab treatment

patients had prolonged progression free survival with pembrolizumab.

Pembrolizumab monotherapy for recurrent glioblastoma was studied in the KEYNOTE-028 trial. [20] Patients were included if they were diagnosed with glioblastoma

Table 3 Clinical Response

\begin{tabular}{ll}
\hline Characteristic & $\begin{array}{l}\text { All evaluable } \\
\text { patients }(n=24)\end{array}$ \\
\hline Best radiographic response & \\
Complete response (CR), no. (\%) & $0(0)$ \\
Partial response (PR), no. (\%) & $2(8)$ \\
Stable disease (SD), no. (\%) & $5(21)$ \\
Progressive disease (PD), no. (\%) & $17(71)$ \\
Median PFS, days (range) & $42(7-282)$ \\
Median OS, days (range) & $121(15-415)$ \\
\hline
\end{tabular}

having PD-L1 expression $\geq 1 \%$, bevacizumab naïve, and unable to receive standard treatment. Median PFS and OS were reported as 2.8 months and 14.4 months, respectively. CheckMate-143 compared nivolumab monotherapy to bevacizumab monotherapy in glioblastoma in patients with first recurrence. Median OS was 9.8 months with nivolumab and 10 months with bevacizumab, PFS was 1.5 months with nivolumab and 3.5 months with bevacizumab, demonstrating no improvement in overall survival. [21] We observed a shorter PFS and OS most likely because patients that failed bevacizumab were also included.

Pembrolizumab was well tolerated in our cohort; toxicities were similar compared to those reported with other malignancies. $[8,9]$ Very few serious adverse events occurred during treatment. Serious adverse events, cerebral edema, seizures and headaches could be related to disease progression or checkpoint inhibition. 


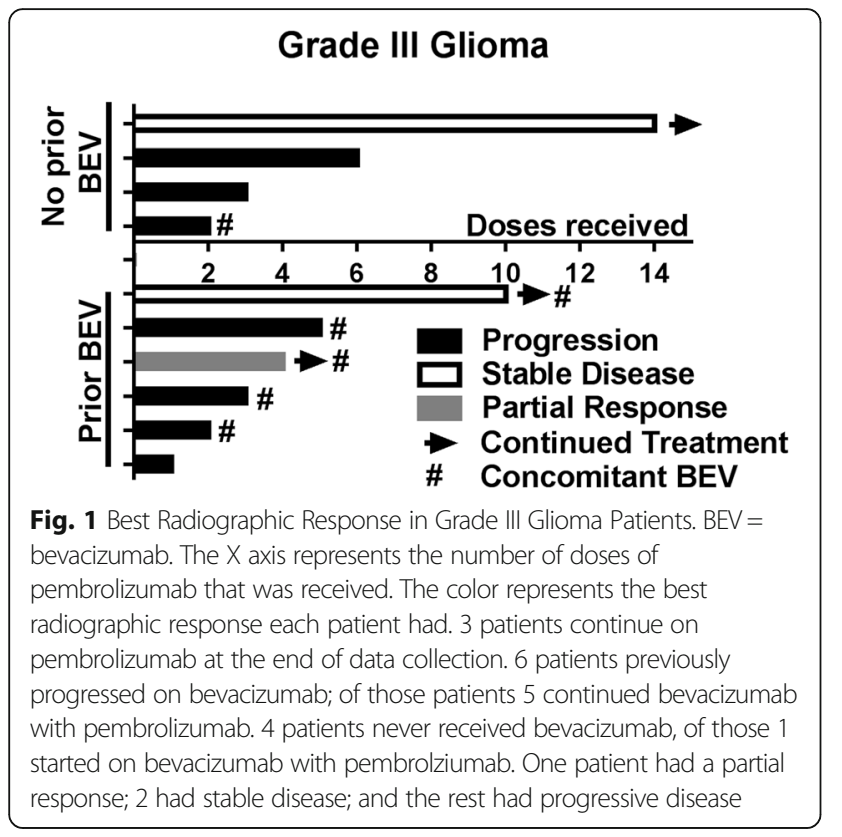

Our study had several limitations. Firstly, it was a retrospective study with a small sample size. Second, many patients received pembrolizumab in combination with other treatment modalities such as bevacizumab, making it difficult to evaluate the effectiveness of pembrolizumab monotherapy in high grade glioma patients. Additionally, we included patients with both WHO grade III and IV gliomas, making it difficult to compare these results to published data that includes only glioblastoma patients. Many of our patients were excluded

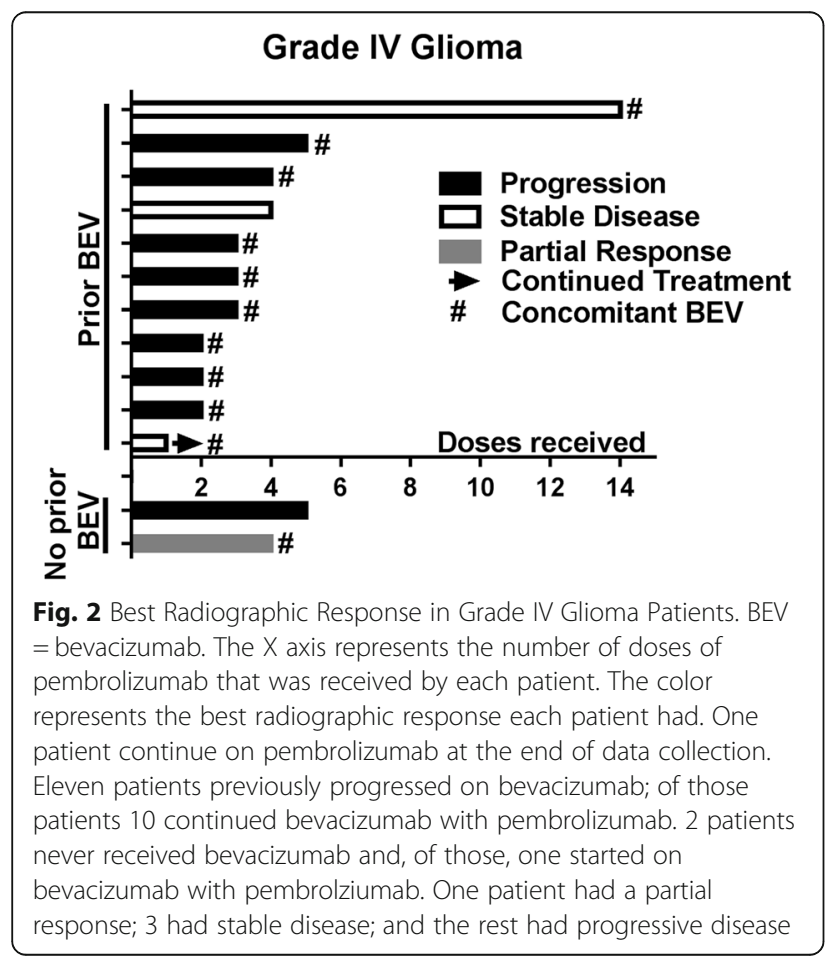

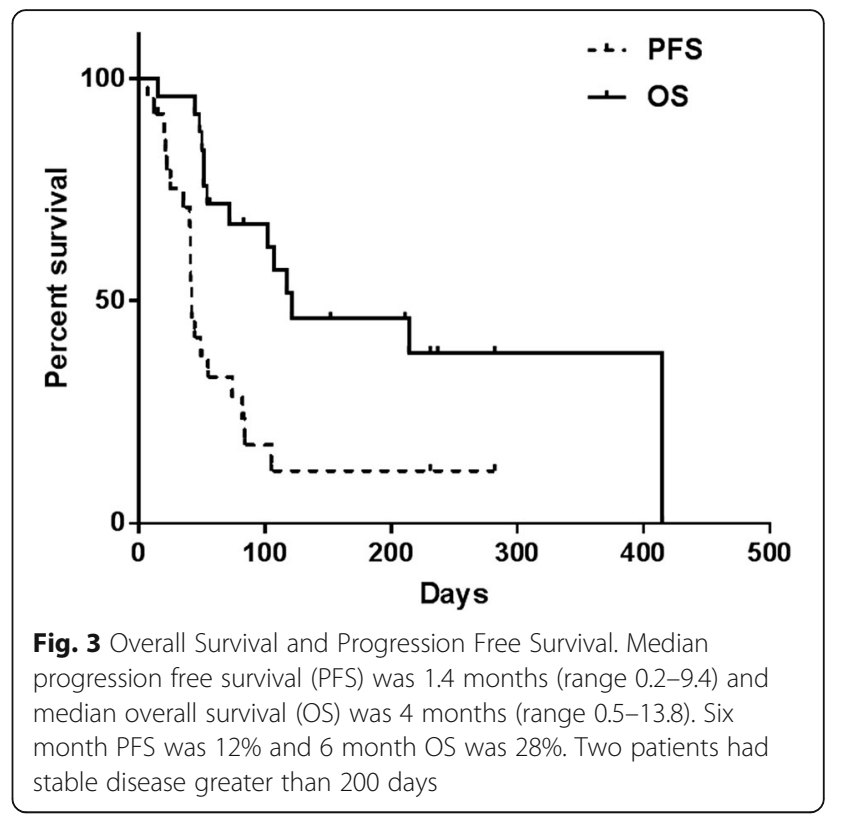

Table 4 Adverse events - incidence and grading according to CTCAE v 4.03

\begin{tabular}{|c|c|c|c|}
\hline Toxicity & $\begin{array}{l}\text { Overall incidence, } \\
\text { no. (\%) }\end{array}$ & $\begin{array}{l}\text { Grade } 1 \text { and } \\
2, \text { no. }(\%)\end{array}$ & $\begin{array}{l}\text { Grade } 3 \text { and } \\
4, \text { no. }(\%)\end{array}$ \\
\hline Hyperglycemia & 19 (79) & $19(79)$ & \\
\hline Fatigue & $19(79)$ & $18(75)$ & $1(4)$ \\
\hline Thrombocytopenia & $12(50)$ & $12(50)$ & \\
\hline Headache & $11(46)$ & $10(43)$ & $1(4)$ \\
\hline Nausea & $10(42)$ & $9(38)$ & $1(4)$ \\
\hline Leukopenia & $9(38)$ & $9(38)$ & \\
\hline ALT elevations & $8(33)$ & $8(33)$ & \\
\hline AST elevations & $7(29)$ & $7(29)$ & \\
\hline Bilirubin elevations & $5(21)$ & $5(21)$ & \\
\hline Neutropenia & $5(21)$ & $5(21)$ & \\
\hline Anemia & $5(21)$ & $4(17)$ & $1(4)$ \\
\hline Seizures & $5(21)$ & $4(17)$ & $1(4)$ \\
\hline Vomiting & $5(21)$ & $4(17)$ & $1(4)$ \\
\hline Thyroid toxicity & $4(17)$ & $4(17)$ & \\
\hline Diarrhea & $4(17)$ & $4(17)$ & \\
\hline Myalgias/Arthralgias & $3(13)$ & $3(13)$ & \\
\hline Rash & $2(8)$ & $2(8)$ & \\
\hline Pyrexia & $2(8)$ & $2(8)$ & \\
\hline Lipase & $1(4)$ & $1(4)$ & \\
\hline Amylase & $1(4)$ & $1(4)$ & \\
\hline Mucositis & $1(4)$ & $1(4)$ & \\
\hline
\end{tabular}


from participation in clinical trials for checkpoint inhibitors due to their WHO grade, previous treatment with bevacizumab, and poor KPS. This patient population differs from previously reported clinical observations using checkpoint inhibitors as it includes grade III and IV gliomas. The observed response rate and survival data might be biased due to the poor prognostics factors in our population (heavily pretreated, bevacizumab-resistance, low KPS performance status). However, these patients are frequently encountered in the clinical setting with little literature to guide treatment decisions.

We also did not account for baseline abnormalities and due to the retrospective nature of this study were unable to differentiate between treatment related toxicity and disease related adverse events. Lastly, we did not assess PD-L1 expression to correlate clinical response to PD-L1 status. Pembrolizumab requires further studies to confirm a benefit for patients with refractory high grade glioma as monotherapy or in combination with chemotherapy or bevacizumab.

\section{Conclusions}

Patients with pathology confirmed refractory high grade gliomas have low response rates to pembrolizumab. However, a small number of patients have a prolonged progression free survival. Pembrolizumab was tolerated with few serious adverse events, even in patients receiving concomitant therapy. Pembrolizumab requires further study to confirm a benefit for patients with refractory high grade glioma as monotherapy or in combination with chemotherapy or bevacizumab.

\footnotetext{
Abbreviations

Bev: Bevacizumab; co-del: 1p19q codeleted; Con Bev: Concomitant bevacizumab; CR: Complete response; CTCAE: Common Terminology Criteria for Adverse Events; HGG: high grade gliomas; intact: 1p19q intact; KPS: Karnofsky performance score; methylated: MGMT methylated; ML: mutational load by MSK impact; MUT: IDH mutant; N: no; N/A: not applicable or unknown; OR: Objective response; ORR: Overall response rate; OS: Overall survival; PD: progressive disease; PD1: Programmed cell death protein-1; PD-L1: Programmed cell death ligand-1; Pembro: pembrolizumab; PFS: Progression free survival; PR: Partial response; Prev Bev: previously progressed on bevacizumab treatment; Pt: Patient; SD: stable disease; unmethylated: MGMT unmethylated; VEGF: vascular endothelial growth factor; WT: IDH wild type; Y: yes
}

\section{Acknowledgements}

not applicable.

\section{Funding}

This study was funded by Memorial Sloan Kettering Cancer Center Support/ Core Grant (P30 CA008748).

\section{Availability of data and materials}

N/A

\section{Authors' contributions}

SNR participated in conceiving the study, obtaining data, interpreting the results, and writing the manuscript; PY participated in obtaining data and reviewing the manuscript; LM participated in obtaining data and reviewing the manuscript; CG participated in conceiving the study, performing statistical analysis, interpreting the results, and writing the manuscript. All authors have read and approved the final manuscript.

\section{Ethics approval and consent to participate}

This study was approved by the Memorial Sloan Kettering Cancer Center Institutional Review Board and consent was waived due to the retrospective nature of this study.

\section{Consent for publication}

N/A

Competing interests

The authors declare that they have no competing interests.

\section{Publisher's Note}

Springer Nature remains neutral with regard to jurisdictional claims in published maps and institutional affiliations.

\section{Author details}

${ }^{1}$ Department of Pharmacy, Memorial Sloan Kettering Cancer Center, 1275 York Ave, New York, NY 10065, USA. ²Department of Neurology, Memorial Sloan Kettering Cancer Center, 1275 York Ave, New York, NY 10065, USA.

Received: 24 July 2017 Accepted: 8 November 2017

Published online: 19 December 2017

References

1. Wen PY, Kesari S. Malignant gliomas in adults. N Engl J Med. 2008:359:492507.

2. Stupp R, Mason WP, van den Bent MJ, Weller M, Fisher B, Taphoorn MJ, Belanger K, Brandes AA, Marosi C, Bogdahn U, et al. Radiotherapy plus concomitant and adjuvant temozolomide for glioblastoma. N Engl J Med. 2005:352:987-96.

3. Lamborn KR, Yung WK, Chang SM, Wen PY, Cloughesy TF, DeAngelis LM, Robins HI, Lieberman FS, Fine HA, Fink KL, et al. Progression-free survival: an important end point in evaluating therapy for recurrent high-grade gliomas. Neuro-Oncology. 2008;10:162-70.

4. Stupp R, Wong ET, Kanner AA, Steinberg D, Engelhard $H$, Heidecke V, Kirson ED, Taillibert S, Liebermann F, Dbaly V, et al. NovoTTF-100A versus physician's choice chemotherapy in recurrent glioblastoma: a randomised phase III trial of a novel treatment modality. Eur J Cancer. 2012;48:2192-202.

5. Friedman HS, Prados MD, Wen PY, Mikkelsen T, Schiff D, Abrey LE, Yung WK, Paleologos N, Nicholas MK, Jensen R, et al. Bevacizumab alone and in combination with irinotecan in recurrent glioblastoma. J Clin Oncol. 2009; 27:4733-40

6. AVASTIN (bevacizumab) [package insert]. Genentech, Inc, San Francisco, CA 2016. [https://www.gene.com/download/pdf/avastin_prescribing.pdf].

7. Chamberlain MC. Salvage therapy with lomustine for temozolomide refractory recurrent anaplastic astrocytoma: a retrospective study. J NeuroOncol. 2015:122:329-38.

8. KEYTRUDA (pembrolizumab) [package insert]. Merck \& Co., Inc. Whitehouse Station, NJ: 2016. [http://www.merck.com/product/usa/pi_circulars/k/ keytruda/keytruda_pi.pdf].

9. OPDIVO (nivolumab) [package insert]. Bristol-Myers Squibb Company, Princeton, NJ; 2017. [http://packageinserts.bms.com/pi/pi_opdivo.pdf].

10. Reck M, Rodríguez-Abreu D, Robinson AG, Hui R, Csőszi T, Fülöp A, Gottfried M, Peled N, Tafreshi A, Cuffe S, et al. Pembrolizumab versus chemotherapy for PD-L1-positive non-small-cell lung cancer. N Engl J Med. 2016;375: 1823-33.

11. Topalian SL, Hodi FS, Brahmer JR, Gettinger SN, Smith DC, McDermott DF, Powderly JD, Carvajal RD, Sosman JA, Atkins MB, et al. Safety, activity, and immune correlates of anti-PD-1 antibody in cancer. N Engl J Med. 2012;366: 2443-54.

12. Berghoff AS, Kiesel B, Widhalm G, Rajky O, Ricken G, Wohrer A, Dieckmann K, Filipits M, Brandstetter A, Weller M, et al. Programmed death ligand 1 expression and tumor-infiltrating lymphocytes in glioblastoma. NeuroOncology. 2015;17:1064-75.

13. Nduom EK, Wei J, Yaghi NK, Huang N, Kong LY, Gabrusiewicz K, Ling X, Zhou S, Ivan C, Chen JQ, et al. PD-L1 expression and prognostic impact in glioblastoma. Neuro-Oncology. 2016:18:195-205.

14. Goldberg SB, Gettinger SN, Mahajan A, Chiang AC, Herbst RS, Sznol M, Tsiouris AJ, Cohen J, Vortmeyer A, Jilaveanu L, et al. Pembrolizumab for patients with melanoma or non-small-cell lung cancer and untreated brain 
metastases: early analysis of a non-randomised, open-label, phase 2 trial. Lancet Oncol. 2016;17:976-83.

15. Roth P, Valavanis A, Weller M. Long-term control and partial remission after initial pseudoprogression of glioblastoma by anti-PD-1 treatment with nivolumab. Neuro-Oncology. 2016;

16. Bouffet E, Larouche V, Campbell BB, Merico D, de Borja R, Aronson M, Durno C, Krueger J, Cabric V, Ramaswamy V, et al. Immune checkpoint inhibition for Hypermutant glioblastoma Multiforme resulting from germline Biallelic mismatch repair deficiency. J Clin Oncol. 2016;34:2206-11.

17. Reardon DA, DeGroot JF, Colman H, Jordan JT, Daras M, Clarke JL, Nghiemphu PL, Gaffey SC, Peters KB. Safety of pembrolizumab in combination with bevacizumab in recurrent glioblastoma (rGBM). J Clin Oncol. 2016;34

18. Ampie L, Woolf EC, Dardis C. Immunotherapeutic advancements for glioblastoma. Front Oncol. 2015;5:12.

19. Garber ST, Hashimoto Y, Weathers SP, Xiu J, Gatalica Z, Verhaak RG, Zhou S, Fuller GN, Khasraw M, de Groot J, et al. Immune checkpoint blockade as a potential therapeutic target: surveying CNS malignancies. Neuro-Oncology. 2016;18:1357-66.

20. TM RDAK, Frenel JS, Santoro A, et al. ATIM-35. Results of the Phase IB KEYNOTE-028 Multi-Cohort Trial of Pembrolizumab Monotherapy in Patietns with Recurrent PD-L1 Positive Glioblastoma Multiforme (GBM). Neuro Oncol. 2016;18:vi25-6.

21. Reardon DA, Omuro A, Brandes AA, et al. Randomized phase 3 study evaluating the efficacy and safety of Nivolumab vs bevacizumab in patients with recurrent glioblastoma: CheckMate 143. Neuro-Oncology. 2017;19:iii21. https:/academic.oup.com/neuro-oncology/article/19/suppl_3/iii21/3743874\#.

\section{Submit your next manuscript to BioMed Central and we will help you at every step:}

- We accept pre-submission inquiries

- Our selector tool helps you to find the most relevant journal

- We provide round the clock customer support

- Convenient online submission

- Thorough peer review

- Inclusion in PubMed and all major indexing services

- Maximum visibility for your research

Submit your manuscript at www.biomedcentral.com/submit

) Biomed Central 